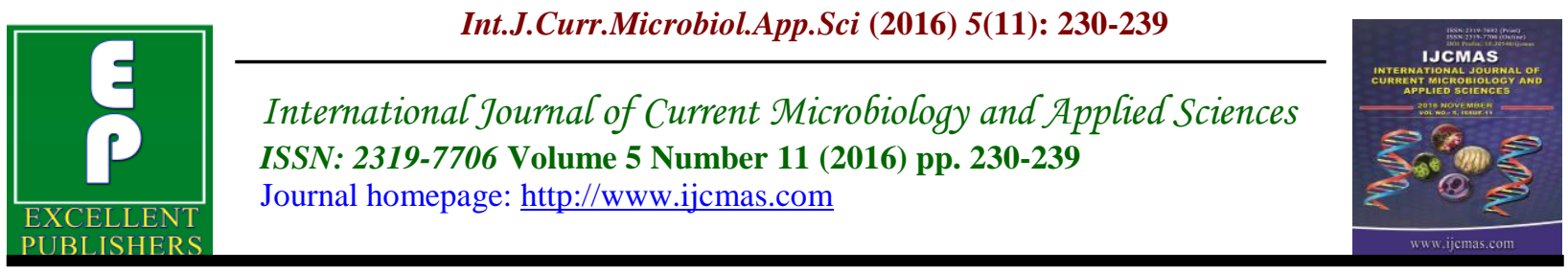

Original Research Article

http://dx.doi.org/10.20546/ijcmas.2016.511.025

\title{
Evaluation of Phenotypic Methods for Detection of Plasmid-Mediated AmpC $\beta$-Lactamases (PMABLs) among Klebsiella pneumoniae
}

\author{
Hala B. Othman and Dalia H. Abd ElHamid* \\ Department of Clinical Pathology, Faculty of Medicine, Ain Shams University, Egypt \\ *Corresponding author
}

Keywords

Plasmid mediated AmpC $\beta$ lactamases (PMABLs) Modified three dimensional test Ceftazidime-Imipenem antagonism test (CIAT) Cefoxitin-Cefotaxime (FOX-CTX) Antagonism.

\section{Article Info}

Accepted:

12 October 2016

Available Online:

10 November 2016

\section{A B S T R A C T}

Enterobacteriaceae producing $\mathrm{AmpC}$ have become a major therapeutic challenge, as they may appear susceptible to expanded-spectrum cephalosporins when initially tested and may lead to therapeutic failure thereafter. Thus, a simple and reliable detection for AmpC producers is needed. Our aim was to assess different phenotypic tests for detection of plasmid mediated Amp C $\beta$ lactamases (PMABLs) in Klebsiella pneumoniae compared to PCR for detection of family specific PMABL genes. Eighty clinical isolates of Klebsiella pneumoniae were screened for AmpC production by cefoxtin (FOX) resistance using disk diffusion test, tested by Cloxacillin Combined Disk Diffusion (CC-DD), Cefoxitin-Cloxacillin DoubleDisk Synergy (CC-DDS), Ceftazidime-Imipenem Antagonism Test (CIAT), and Cefoxitin-Cefotaxime (FOX-CTX) Antagonism. PMABLs were detected in 26 $(32.5 \%)$ out of 80 isolates. $40(50 \%)$ of 80 isolate were resistant to cefoxitin with (84.6\%) sensitivity, 26 (32.5\%) were positive CC-DD with (61.5\%) sensitivity; 22 (27.5\%) were positive (CC-DDS) with (38.5\%) sensitivity; 4 (5\%) were positive (CIAT) $6(10 \%)$ were positive (FOX- CTX). In conclusion, CC-DD is a simple relatively sensitive and specific test for detection of PMABLs. CIAT and FOXCTX antagonism tests are simple reliable tests for detecting the induced PMABLs.

\section{Introduction}

Ambler class $\mathrm{C}$ or (AmpC) $\beta$-lactamases production is one of the mechanisms of resistance to $\beta$-lactam antibiotics in Enterobacteriaceae conferring resistance to a wide variety of $\beta$-lactam antibiotics including penicillins, 7- $\alpha$-methoxy cephalosporins, oxyimino cephalosporins, monobactam. These organisms are not inhibited by clavulanic acid (Mohamudha et al., 2012), but their susceptibility to cefepime and cefpirome is minimally affected and is unaffected for carbapenems (Seral et al., 2012).
AmpC $\beta$-lactamases are typically encoded on the chromosome of many Gram-negative bacteria including Citrobacter, Serratia, Enterobacter species where its expression is usually inducible. It may also occur in Escherichia coli (E. coli) although it is not usually inducible (Philipon et al., 2002). On the other hand, Klebsiella isolates does not possess chromosomal AmpC (Pérez-Pérez and Hanson, 2002).

Plasmid mediated AmpC $\beta$-lactamases (PMABLs) genes are non inducible 
(Mohamudha et al., 2012). They can appear in bacteria lacking or poorly expressing chromosomal AmpC genes as E. coli, Klebsiella pneumonia and Proteus mirabilis (Seral et al., 2012). The commonly reported genotypes are ACC, FOX, MOX, DHA, CIT and EBC (Manoharan et al., 2012).

The prevalence of infection with organisms carrying PMABLs varies depending on the type of enzyme and geographical location (Seral et al., 2012). The majority of plasmidmediated AmpC genes are found in nosocomial isolates of E. coli and Klebsiella pneumoniae which are responsible for nosocomial infection and colonization (Black et al., 2005). These organisms are often associated with multidrug resistance, leaving a few therapeutic options (Mohamudha et al., 2012). So, the accurate detection of PMABL-producing organisms is crucial for controlling their spread and provide the optimal treatment option (PérezPérez and Hanson, 2002).

There are no Clinical Laboratory Standards Institute (CLSI) guidelines available for optimal AmpC detection and confirmation, Hence, genotypic characterization is considered as the gold standard (Mohamudha et al., 2012). However, they are expensive and not available in all institutions as routine tests. So, various phenotypic methods have been developed such as AmpC disk test (Mohamudha et al., 2012), modified double disk test (Singhal et al., 2005), cefoxitin-cloxacillin double-disk synergy test (CC-DDS) (Polsfuss et al., 2011), modified three dimensional test (Shahid et al., 2004), cefoxtin agar medium method (Nasim et al., 2004), agar dilution method using cefoxitin with and without added cloxacillin (Tan et al., 2009) and Etest AmpC (Polsfuss et al., 2011).

Our aim was to assess different phenotypic tests for detection of PMABL in Klebsiella pneumoniae isolates compared to multiplex PCR for detection of PMABL genes.

\section{Materials and Methods}

This study was conducted on 80 clinical isolates of Klebsiella pneumoniae recovered from different clinical specimens referred to Central Microbiology Laboratory of Ain Shams University Hospitals from September 2012 to February 2013.

All Isolates were subjected to the following:

\section{Cefoxitin disk diffusion (DD) test}

For screening of Amp C production by using cefoxtin (FOX) 30 $\mu \mathrm{g}$ disk (Polsfuss et al., 2011). Isolates showing an inhibition zone diameter of $\leq 14 \mathrm{~mm}$ were considered resistance (CLSI, 2012) (Figure 1).

\section{Cloxacillin Combined Disk Diffusion Test (CC-DD) (Tan et al., 2009):}

For detection of AmpC production

a-Preparation of stock solution of Cloxacillin (CLOX): $20 \mathrm{mg}$ of CLOX powder (1g) (Sigma Aldrich, Singapore) was dissolved in two milliliter of sterile distilled water $(10 \mathrm{mg} / \mathrm{ml})$.

b- Preparation of disks containing cloxacillin: $20 \mu \mathrm{L}$ of stock solution containing CLOX was dispensed onto cefoxitin (FOX) disk $(30 \mu \mathrm{g})$. The final concentration of CLOX on the disk was $200 \mu \mathrm{g}$. Cefoxtin disks containing CLOX were allowed to dry for 15 mins and were used within 60 mins.

c- Interpretation: $A \geq 4 \mathrm{~mm}$ increased difference in FOX-CLOX combined disk inhibition zones compared to FOX disk alone was indicative for $\mathrm{AmpC}$ production (Figure 1). 
Cefoxitin-Cloxacillin Synergy Test (CC-DDS) (Ruppé et al., 2006)

\section{For detection of AmpC production}

a- Preparation of stock solution of CLOX: $19.5 \mathrm{mg}$ of CLOX powder was dissolved in two millilitre of sterile distilled water $(9.75$ $\mathrm{mg} / \mathrm{ml})$.

b- Preparation of disks containing cloxacillin: $20 \mu \mathrm{L}$ of stock solution containing CLOX $(195 \mu \mathrm{g})$ was dispensed onto $5 \mu \mathrm{g}$ cloxacillin disk (OB) (Oxoid, UK). The final concentration of cloxacillin on the disk was $200 \mu \mathrm{g}$. Cloxacillin disks were allowed to dry for 15 mins and were used within 60 mins. The disk was put at a distance of $10 \mathrm{~mm}$ edge to edge from FOX disk.

c- Interpretation: A keyhole or ghost zone (synergism) between CLOX and FOX disks indicates the presence of an $\mathrm{AmpC} \beta$ lactamase (Figure 1).

Ceftazidime-Imipenem Antagonism Test (CIAT) (Cantarelli et al., 2007) and Cefoxitin-Cefotaxime (FOX-CTX) Antagonism Test (Upadhyay et al., 2010)

Antagonism, indicated by (D shaped); a blunted inhibition zone around CAZ disk adjacent to IPM disk (Figure 2), or CTX disk adjacent to FOX disk (Figure 3), at a distance of $20 \mathrm{~mm}$ from center to center. This was regarded as positive for inducible AmpC $\beta$ lactamase production.

Double Disk Synergy Test (DDS) for detection of ESBLs (Coudron et al., 2003)

Ceftazidime $(30 \mu \mathrm{g})$, ceftriaxone $(30 \mu \mathrm{g})$, or cefpdoxime $(10 \mu \mathrm{g})$, were placed $15 \mathrm{~mm}$ (edge to edge) from augmentin (amoxicillin / clavulanate)

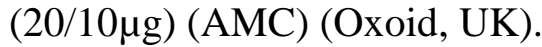

Detection of family specific plasmidmediated $A m p C$ genes (ACC, FOX, MOX, DHA, CIT and EBC) by Multiplex-PCR (Pérez-Pérez and Hanson, 2002).

Bacterial DNA was extracted using DNA extraction kit (Jena Bioscience, Germany). The reaction mixture included KAPA2 $\mathrm{G}^{\mathrm{TM}}$ Fast PCR master mix (KAPA BIOSYSTEM, USA), Oligonucleotide primers (Sigma, Germany) according to (Pérez-Pérez and Hanson, 2002) (table 1) in a final volume of $25 \mu \mathrm{l}$. Well-characterized clinical isolates for PMABL genes were used as positive controls and nuclease free water as a negative control.

The amplification program was started by initial denaturation for two minutes at $95^{\circ} \mathrm{C}$, 30 cycles of amplification consisting of: Denaturation at $95^{\circ} \mathrm{C}$ for $15 \mathrm{sec}$., annealing at $65^{\circ} \mathrm{C}$ for $30 \mathrm{sec}$., extension at $72^{\circ} \mathrm{C}$ for 30 sec., and final extension at $72^{\circ} \mathrm{C}$ for 7 minutes. The PCR products were analyzed by 3\% agarose gel (Sigma, Germany) electrophoresis and bands were visualized with UV light after staining with ethidium bromide (Promega, USA). Images were captured on a Kodak Camera (Japan). The molecular size marker (Promega, USA), gave different bands ranging from 100bp1000bp (Promega, USA).

\section{Statistical analysis}

Data were presented and suitable analysis was done according to the type of data obtained for each parameter.

A. Descriptive statistics: Frequency and percentage of non numerical data.

B. Analytical statistics:

The diagnostic test evaluation; sensitivity, specificity and the positive and negative 
predictive values and. All the analyses were performed with commercially available software (SPSS 15.0.1 for windows; SPSS Inc, Chicago, IL, USA, 2001).

\section{Results and Discussion}

Plasmid mediated AmpC $\beta$ lactamases (PMABLs) genes were detected in 26 (32.5\%) of the 80 tested isolates. Out of 26 PCR-positive isolates, $20 \quad(76.9 \%)$ were cefoxtin resistant and; $16(61.5 \%)$ were positive by CC-DD test; $10(38.5 \%)$ were positive by CC-DDS test and ; 2 (7.7\%) were CIAT positive; 4 (15.4\%) were (FOXCTX) antagonism test positive. Sixteen out of $26(61.5 \%)$ isolates produced AmpC only and $10 / 26 \quad(38.5 \%)$ isolates were AmpC/ESBL co-producers .

On the other hand, among 54 PCR-negative isolates, $20(37.0 \%)$ isolates were cefoxtin resistant and 34(63.0\%) were cefoxtin sensitive; $10(18.5 \%)$ were positive by CCDD test; $12(22.2 \%)$ were positive by CCDDS test; 2 ( $3.7 \%$ ) were CIAT positive; 2 ( $3.7 \%$ ) were CTX- FOX antagonism test positive. $40 / 54(74.1 \%)$ isolates were only ESBL producers by DDS test and 14/54 (25.9\%) isolates were non AmpC/non ESBL producers.

Detection of plasmid-mediated AmpC genes (ACC, FOX, MOX, DHA, CIT and EBC) by Multiplex-PCR

Among 26 PCR-positive isolates, 16 $(61.5 \%)$ of isolates had MOX gene, 16 $(69.2 \%)$ of isolates had CIT gene, 10 $(38.5 \%)$ of isolates had ACC gene, 10 $(38.5 \%)$ of isolates had FOX gene, two $(7.7 \%)$ of isolates had DHA gene. EBC gene was detected in two isolates. The distribution of the genes per isolate is demonstrated in (table 3).
Screening for AmpC production by Fox disk diffusion method (DD)

In our study, Cefoxitin (DD) has a relatively good sensitivity as a screening test for PMABLs production; as 20 out of 26 PCR positive isolates were cefoxtin resistant $(<14 \mathrm{~mm})$ with a sensitivity of $76.9 \%$ and a specificity of $63.0 \%$ for detection of PMABLs. Eighteen (45\%) out of 40 cefoxitin resistant isolates were carrying PMBLs genes.

While, Upadhyay et al., (2011) demonstrated that only $27 / 309(8.7 \%)$ of cefoxitin resistant isolates $(<18 \mathrm{~mm})$ were confirmed positive for AmpC gene by multiplex PCR.

Ingram et al., (2011) demonstrated that 72/74 PCR-positive Enterobacteriaceae isolates showed resistance to cefoxitin with a sensitivity of $97 \%$, and a specificity of $64 \%$.

Also, Polsfuss et al., (2011) found that the sensitivity of cefoxitin for the detection of AmpC production compared to PCR was $97.4 \%$ and the specificity was $78.7 \%$.

On the other hand, Peter-Getzlaff et al., (2011) demonstrated that 21/51 (41\%) E. coli isolates were considered true AmpC producers by PCR. Cefoxitin resistance was found to be a discriminative parameter, detecting 20/21 (95.2\%) AmpC-producing strains.

In our study, 6/26 (23.1\%) PCR-positive isolates were carrying plasmid encoded AmpC $\beta$-lactamases of the family ACC rendering these strains appearing cefoxitin susceptible; as Peter-Getzlaff et al., (2011) stated that ACC-1 is inhibited by cefoxitin; so, FOX may appear susceptible. 
The 20 isolates that were cefoxitin resistant in the AmpC-negative isolates could be due to porin channel alterations and mutations in these isolates. Also, cefoxitin may be a substrate to active efflux pump in clinical isolates (Fam et al., 2013).

\section{Cloxacillin combined disk diffusion test (CC-DD)}

Cloxacillin combined disk diffusion test (CC-DD) was positive in 16/ 26 PCR positive isolates with $61.5 \%$ sensitivity and $81.5 \%$ specificity for detection of PMABL production when using cloxacillin concentration of $200 \mu \mathrm{g} / \mathrm{disk}$ and $\mathrm{a} \geq 4 \mathrm{~mm}$ increase in zone diameter as a cutoff.

However, Tan et al., (2009) demonstrated that using of CC-DD test accurately detected the majority of AmpC-positive isolates with best sensitivity and specificity; both (95\%) when using $\mathrm{a} \geq 4 \mathrm{~mm}$ increase in zone diameter as a cutoff and $200 \mu \mathrm{g}$ as cloxacillin concentration on 255 clinical isolates of Enterobacteriaceae. Similarly, Polsfuss et al., (2011) found that the sensitivity for CCDD test for detection of AmpC was 97.2\% and the specificity was $100 \%$ when using the same concentration and the same cutoff compared to both Modified three dimensional test and PCR.

Also, Peter-Getzlaff et al., (2011) demonstrated that CC-DD test correctly detected 20/21 (95.2\%) AmpC (chromosomal and plasmid- mediated) positive E. coli strains by PCR and did not give false-positive results when using the same concentration and the same cutoff.

Ingram et al., (2011) demonstrated that the sensitivity and specificity of CC-DD test when using cloxacillin $(100 \mu \mathrm{g})$ were $53 \%$ and $99 \%$ respectively for detection of AmpC production. The sensitivity and specificity were improved to $(70 \%$ and $100 \%)$ respectively when using cloxacillin $(200 \mu \mathrm{g})$.
On the other hand, Giske et al., (2007) referred some of the detection failures to very high levels of AmpC when using a $\geq$ $5 \mathrm{~mm}$ increase in zone diameter as a cutoff and $750 \mu \mathrm{g}$ as cloxacillin concentration. So, they suggested that the detection of AmpC production could possibly be improved by increasing the concentration of cloxacillin.

However, the false-negative results in our study may be explained by the presence of some isolates co-producing both AmpC and ESBL. This combination is known to weaken the performance of cloxacillin for AmpC detection (Ingram et al., 2011).

On the other hand, in our study, 10/54 $(18.5 \%)$ PCR negative isolates were positive by CC-DD test.

\section{Cefoxitin-cloxacillin double disk senergy test (CC-DDS)}

Cefoxitin-cloxacillin double disk senergy test (CC-DDS) was positive in 10 out of 26 PCR-positive isolates with low sensitivity $38.5 \%$ however, a relatively high specificity $77.7 \%$ for detection of AmpC production.

Fam et al., (2013) found that CC-DDS test with cloxacillin concentration of $200 \mu \mathrm{g}$ had more sensitivity $(82.4 \%)$ and specificity $(93 \%)$ for detection of PMABL production in Klebsiella spp compared to PCR. But, Gude et al., (2012) demonstrated a high sensitivity $96 \%$ and a low specificity $29 \%$ of CC-DDS test for detection of isolates harbored a plasmid-mediated AmpC gene compared to PCR when using commercially available cloxacillin disks with concentration of $500 \mu \mathrm{g}$.

On the other hand, in our study, 12/54 (22.2\%) PCR negative isolates, were positive by CC-DDS test. The false positive isolates by both CC-DD and CC-DDS tests may be explained by the possibility of 
presence of more $A m p C \beta$-lactamase genes that continue to expand beyond those contained in the six genes families covered by PCR (Fam et al., 2013).

Plasmid-mediated AmpC $\quad \beta$-lactamases (PMABLs) are almost always non inducible. However, some carry inducible plasmidmediated AmpC gene such as CMY (one of the CIT family) and DHA (Mirelis et al., 2006).

In our study, the inducibility of PMABLs were tested by ceftazidime-imipenem antagonism test (CIAT) and cefoxitincefotaxime (FOX-CTX) antagonism test in which imipenem and cefoxitin respectively were used as inducers.

\section{Ceftazidime-Imipenem Antagonism Test (CIAT)}

It was positive in two $(7.7 \%)$ out of $26 \mathrm{PCR}$ positive isolates. These isolates were carrying CIT gene. Cantarelli et al. (2007) found that CIAT detected chromosomal AmpC more perfectly than PMABLs as $100 \%$ of isolates were induced by imipenam, two (6\%) were carrying plasmid (blaDHA) and 32 (94\%) isolates were chromosomal. However, in our study there were 16 isolates that were carrying CIT gene, but were negative by the test; These islolates were resistance to ceftazidime and were having other Amp C genes which may inhibit or mask the effect of the inducer (imipenem).

Also, Cantarelli et al., (2007) stated that, CIAT test can not be used to test strains showing no inhibition zone for ceftazidime or for those strains bearing plasmidmediated AmpC enzymes that are not typically inducible.

\section{Cefoxitin-Cefotaxime Antagonism}

(FOX-CTX)

It was positive in four (15.4\%) out of 26 PCR positive isolates; the two isolates with the CIT gene and the two isolates with DHA gene. On the other hand, both CIAT and FOX-CTX was positive in two (3.7\%) PCR negative isolates. This may be due to other mechanism of resistance that could be induced by these two inducers.

Table.1 Primar sequences of PMABL genes (Pérez-Pérez and Hanso, 2002).

\begin{tabular}{|c|c|c|c|c|c|c|c|c|c|c|}
\hline & Primer & & & equens & $5^{\prime}$ to $3^{\prime}$ & syntl & zed) & & & Bp \\
\hline 1 & ACCMF & AAC & $\mathrm{AGC}$ & CTC & $\mathrm{AGC}$ & AGC & CGG & TTA & & \multirow{2}{*}{346} \\
\hline 1 & ACCMR & TTC & $\mathrm{GCC}$ & GCA & ATC & ATC & $\mathrm{CCT}$ & AGC & & \\
\hline 2 & EBCMF & TCG & GTA & AAG & $\mathrm{CCG}$ & ATG & TTG & CGG & & \multirow{2}{*}{302} \\
\hline 2 & EBCMR & CTT & $\mathrm{CCA}$ & CTG & CGG & CTG & $\mathrm{CCA}$ & GTT & & \\
\hline 3 & FOXMF & AAC & ATG & GGG & TAT & CAG & GGA & GAT & $\mathrm{G}$ & \multirow{2}{*}{190} \\
\hline 3 & FOXMR & CAA & AGC & GCG & TAA & $\mathrm{CCG}$ & GAT & TGG & & \\
\hline 4 & DHAMF & AAC & TTT & CAC & $\mathrm{AGG}$ & TGT & GCT & GGG & $\mathrm{T}$ & \multirow{2}{*}{405} \\
\hline 4 & DHAMR & $\mathrm{CCG}$ & TAC & GCA & TAC & TGG & CTT & TGC & & \\
\hline 5 & CITMF & TGG & $\mathrm{CCA}$ & GAA & CTG & $\mathrm{ACA}$ & GGC & AAA & & \multirow{2}{*}{462} \\
\hline 5 & CITMR & TTT & CTC & CTG & $\mathrm{AAC}$ & GTG & GCT & GGC & & \\
\hline 6 & MOXMF & GCT & GCT & CAA & GGA & GCA & $\mathrm{CAG}$ & GAT & & \multirow{2}{*}{520} \\
\hline 6 & MOXMR & CAC & ATT & GAC & ATA & GGT & GTG & GTG & $\mathrm{C}$ & \\
\hline
\end{tabular}


Table.2 The results of all tests in 80 isolates

\begin{tabular}{|c|c|c|c|}
\hline \multicolumn{2}{|l|}{ Test } & $\mathbf{N}()$ & $\%$ \\
\hline \multirow{2}{*}{$\begin{array}{l}\text { Cefoxtin Disk Diffusion (DD) } \\
\text { Method }\end{array}$} & Sensitive & 40 & 50 \\
\hline & Resistant & 40 & 50 \\
\hline \multirow{2}{*}{$\begin{array}{c}\text { Cloxacillin Combined Disk Diffusion } \\
\text { Test (CC-DD) }\end{array}$} & Positive & 26 & 32.5 \\
\hline & Negative & 54 & 67.5 \\
\hline \multirow{2}{*}{ Cefoxitin-Cloxacillin Double Disk Senergy Test (CC-DDS) } & Positive & 22 & 27.5 \\
\hline & Negative & 58 & 72.5 \\
\hline \multirow{2}{*}{ Ceftazidime-Imipenem Antagonism Test (CIAT) } & Positive & 4 & 5.0 \\
\hline & Negative & 76 & 95.0 \\
\hline \multirow{2}{*}{$\begin{array}{c}\text { Cefoxitin-Cefotaxime (FOX-CTX) } \\
\text { Antagonism Test }\end{array}$} & Positive & 6 & 7.5 \\
\hline & Negative & 74 & 92.5 \\
\hline \multirow{2}{*}{$\begin{array}{l}\text { Double Disk Senergy Test (DDS) for } \\
\text { ESBL Detection }\end{array}$} & Positive & $\begin{array}{c}50 \\
(62.5 \%)\end{array}$ & 62.5 \\
\hline & Negative & 30 & 37.5 \\
\hline \multirow{2}{*}{ PCR } & Positive & 26 & 32.5 \\
\hline & Negative & 54 & 67.5 \\
\hline
\end{tabular}

Table.3 The distribution of PMABLs genes among 26 PCR-positive isolates (Per isolate).

\begin{tabular}{|c|c|c|}
\hline \multicolumn{2}{|c|}{ PMABLs genes } & $\mathbf{N}(\%)$ \\
\hline \multirow{4}{*}{ Positive } & $26(32.5 \%)$ \\
\cline { 2 - 3 } & ACC & $6(7.5 \%)$ \\
\cline { 2 - 3 } & CIT/MOX & $4(5.0 \%)$ \\
\cline { 2 - 3 } & CIT & $2(2.5 \%)$ \\
\cline { 2 - 3 } & CIT/MOX/FOX & $2(2.5 \%)$ \\
\cline { 2 - 3 } & FOX/CIT/MOX & $2(2.5 \%)$ \\
\cline { 2 - 3 } & CIT/FOX/ACC & $2(2.5 \%)$ \\
\cline { 2 - 3 } & FOX/CIT/MOX & $2(2.5 \%)$ \\
\cline { 2 - 3 } & MOX & $6(7.5 \%)$ \\
\cline { 2 - 3 } & DHA & $2(2.5 \%)$ \\
\cline { 2 - 3 } & ACC/CIT/FOX/EBC & $2(2.5 \%)$ \\
\hline
\end{tabular}


Fig.1 (a) Cefoxitin disk resistance using DD method. (b) CC-DDS test. Positive synergism between CLOX (OB) $(200 \mu \mathrm{g})$ and FOX disks. (c) CC-DD test. $\geq 4 \mathrm{~mm}$ increased difference in the FOX-CLOX combined disk inhibition zones compared to FOX disk alone.

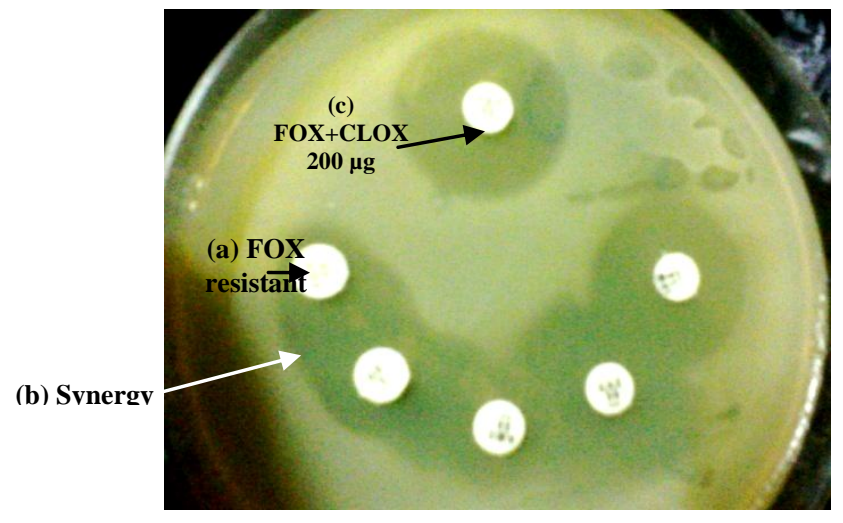

Fig.2 CIAT. Positive antagonism (D shaped) around CAZ disk adjacent to IPM disk.

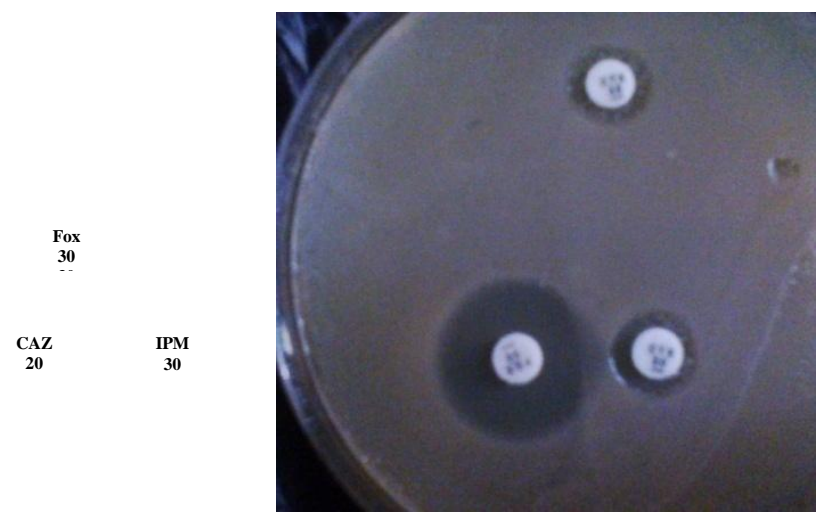

Fig.3 Cefoxitin-Cefotaxime Antagonism Test. Positive antagonism (D shaped) around CTX disk adjacent to FOX disk.

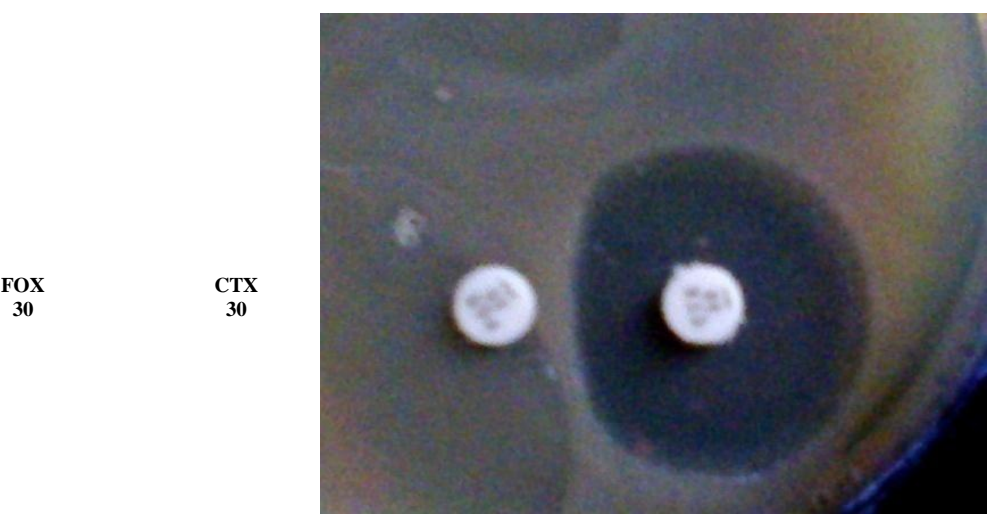

In conclusion, CC-DD is a simple relatively sensitive and specific test for detection of PMABLs. CIAT and FOX- CTX antagonism tests are simple reliable tests for detecting the induced PMABLs in Klebsiella pneumoniae. 


\section{References}

Black, A.J., Moland, S.E. and Thomson, S.K. 2005. AmpC Disk Test for Detection of Plasmid-Mediated AmpC $\beta$-Lactamases in Enterobacteriaceae Lacking Chromosomal AmpC $\beta-$ Lactamases. J. Clin. Microbiol., 43 (7): r3110-3113.

Cantarelli, V.V., Inamine, E., Brodt, T.C.Z., Secchi, C., Cavalcante, B.C. and Pereira, F.S. 2007. Utility of the Ceftazidime-Imipenem Antagonism Test (CIAT) to Detect and Confirm the Presence of Inducible AmpC BetaLactamases Among Enterobacteriaceae. The Brazilian J. Infect. Dis., 11(2): 237-239.

Clinical and Laboratory Standards Institute (CLSI). 2012. Performance standards for antimicrobial susceptibility testing. Approved Standard, M02-A9. Wayne, PA: Clinical and Laboratory Standards Institute.

Coudron, P.E., Hanson, N.D., Climo, M.W. 2003. Occurrence of extended spectrum and AmpC beta-lactamases in bloodstream isolates of Klebsiella pneumoniae: isolates harbor plasmidmediated FOX- 5 and ACT-1 AmpC beta-lactamases. J. Clin. Microbiol., 41: 772-777.

Fam, N., Gamal, D., El Said, M., Abo-Fadi, L., El-Dabei, E., El-Attar, S., Sorur, A., Fouad, S. and Klena, J. 2013. Detection of Plasmid-Mediated AmpC Beta-Lactamases in Clinically Significant Bacterial Isolates in a Research Institute Hospital in Egypt. Life Sci. J., 10(2): 2294-2304.

Giske, G.C., Haldorsen, B., Lundblad, E.W., Aasnaes, B., Bylund, L., Ho, P., Simonsen, G.S., Yousef, A. and Sundsfjord, A. 2007. Phenotypic Detection of AmpC: Comparison of Etest AmpC Strips and Disk Synergy
Assays with Cloxacillin, Boronic Acid and EDTA. Karolinska InstitutetMTC, Department of Clinical Micobiology.

Gude, M.J., Seral, C., Sáenz, Y., GonzalezDominguez, M., Torres, C. and Castillo, F.J. 2012. Evaluation of four phenotypic methods to detect plasmidmediated AmpC $\beta$-lactamases in clinical isolates. Eur. J. Clin. Microbiol. Infect. Dis.; 31: 2037-2043. Ingram P.R., Inglis T.J.J., Vanzetti T.R., Henderson B.A., Harnett G.B. and Murray R.J. (2011): Comparison of methods for AmpC $\beta$-lactamase detection in Enterobacteriaceae. $J$. Med. Microbiol., 60: 715-721.

Manoharan, A., Sugumar, M., Kumar, A., Jose, H. and Mathai, D. 2012. Phenotypic and molecular characterization of AmpC $\beta$ lactamases among Escherichia coli, Klebsiella spp. and Enterobacter spp. From five Indian medical centers. Indian J. Med. Res., 135: 359-364.

Mirelis, B., Rivera, A., Miró, E., Mesa, R.J., Navarro, F. and Coll, Y.P. 2006. A simple phenotypic method for differentiation between acquired and chromosomal AmpC $\beta$-lactamases in Escherichia coli. Enferm. Infecc. Microbiol. Clin., 24(6): 370-372.

Mohamudha, P.R., Harish, B.N. and Parija, S.C. 2012. Molecular description of plasmid-mediated AmpC $\beta$-lactamases among nosocomial isolates of Escherichia coli \& Klebsiella pneumoniae from six different hospitals in India. Indian J. Med. Res., 153: 114-119.

Nasim, K., Elsayed, S., Pitout, J.D.D., Conly, J., Church, D.L. and Gregson, D.B. 2004. New Method for Laboratory Detection of AmpC $\beta$ Lactamases in Escherichia coli and Klebsiella pneumoniae. J. Clin. 
Microbiol., 42: 4799-4802.

Pérez-Pérez, F.J. and Hanson, N.D. 2002. Detection of Plasmid-Mediated AmpC $\beta$-Lactamase Genes in Clinical Isolates by Using Multiplex PCR J. Clin. Microbiol., 40 (6): 2153-2162.

Peter-Getzlaff, S., Polsfuss, S., Poledica, M., Hombach, M., Giger J., Bottger E.C., Zbinden, R. and Bloemberg G. V. 2011. Detection of AmpC BetaLactamase in Escherichia coli: Comparison of Three Phenotypic Confirmation Assays and Genetic Analysis. J. Clin. Microbiol., 498. 2924-2932.

Philippon, A., Arlet, B. and Jacoby, G.A. 2002. Plasmid-determined AmpC-type $\beta$-lactamases. Antimicrob. Agents Chemother., 46: 1-11.

Polsfuss, S., Bloemberg, G.V., Giger, J., Meyer, V., Bottger, E.C. and Hombach, M. 2011. Practical Approach for Reliable Detection of AmpC Beta-Lactamase-Producing Enterobacteriaceae. J. Clin. Microbiol., 49: 2798-2803.

Ruppé, E., Bidet, P., Verdet, C., Arlet, G. and Bingen, E. 2006. First Detection of the Ambler Class C 1 AmpC $\beta$ Lactamase in Citrobacter freundii by a New Simple Double-Disk Synergy Test. J. Clin. Microbiol., 44 11. 42044207.

Seral, C., Gude, M. and Castillo, F.J. 2012. Emergence of plasmid mediated AmpC $\quad \beta$-lactamases: origin, importance, detection and therapeutical options. Rev. Esp. Quimioter., 52. 89-99.
Shahid, M., Malik, A., Agrawal, M. and Singhal, S. 2004. Phenotypic detection of extended-spectrum and AmpC $\beta$ lactamases by a new spot-inoculation method and modified threedimensional extract test: comparison with the conventional threedimensional extract test. $J$. Antimicrob. Chemother., 54: 684-687.

Singhal, S., Mathur, T., Khan, S., Upadhyay, D.J., Chugh, S., Gaind, R. and Rattan A. 2005. Evaluation of methods for AmpC $\beta$-lactamase in gram negative clinical isolates from tertiary care hospitals. Indian J Med Microbiol. 23 2.120-124.

Tan, T.Y., He, J., Yong, Ng L.S., Koh, T.H. and Hsu, L.Y. 2009. Evaluation of Screening Methods to Detect PlasmidMediated AmpC in Escherichia coli, Klebsiella pneumonia and Proteus mirabilis. Antimicrob. Agents Chemother., 53: 146-149.

Upadhyay, S., Sen, M.R., and Bhattacharjee, A. 2010. Presence of different betalactamase classes among clinical isolates of Pseudomonas aeruginosa expressing AmpC beta-lactamase enzyme. J. Infect. Dev. Ctries., 44. 239-242.

Upadhyay S., Sen, M.R., Bhattacharjee, A., Prakash, P., Bajpai, R.C. and Anupurba, S. 2011. Diagnostic utility of combination of inducer and inhibitor based assay in detection of Pseudomonas aeruginosa producing AmpC $\beta$-lactamase. J. Microbiol. Methods, 87: 116-118.

\section{How to cite this article:}

Hala B. Othman and Dalia H. Abd ElHamid. 2016. Evaluation of Phenotypic Methods for Detection of Plasmid-Mediated AmpC $\beta$-Lactamases (PMABLs) among Klebsiella pneumoniae. Int.J.Curr.Microbiol.App.Sci. 5(11):230-239.

doi: http://dx.doi.org/10.20546/ijcmas.2016.511.025 